\title{
On winning fast in Avoider-Enforcer games
}

\author{
János Barát* \\ Department of Computer Science and Systems Technology \\ University of Pannonia, Egyetem u. 10, 8200 Veszprém, Hungary \\ barat@dcs.vein.hu \\ Miloš Stojaković ${ }^{\dagger}$ \\ Department of Mathematics and Informatics \\ University of Novi Sad, Serbia \\ milos.stojakovic@dmi.uns.ac.rs
}

Submitted: Oct 22, 2009; Accepted: Mar 27, 2010; Published: Apr 5, 2010

Mathematics Subject Classifications: 91A43, 91A24

\begin{abstract}
We analyze the duration of the unbiased Avoider-Enforcer game for three basic positional games. All the games are played on the edges of the complete graph on $n$ vertices, and Avoider's goal is to keep his graph outerplanar, diamond-free and $k$-degenerate, respectively. It is clear that all three games are Enforcer's wins, and our main interest lies in determining the largest number of moves Avoider can play before losing.

Extremal graph theory offers a general upper bound for the number of Avoider's moves. As it turns out, for all three games we manage to obtain a lower bound that is just an additive constant away from that upper bound. In particular, we exhibit a strategy for Avoider to keep his graph outerplanar for at least $2 n-8$ moves, being just 6 short of the maximum possible. A diamond-free graph can have at most $d(n)=\left\lceil\frac{3 n-4}{2}\right\rceil$ edges, and we prove that Avoider can play for at least $d(n)-3$ moves. Finally, if $k$ is small compared to $n$, we show that Avoider can keep his graph $k$-degenerate for as many as $e(n)$ moves, where $e(n)$ is the maximum number of edges a $k$-degenerate graph can have.
\end{abstract}

\footnotetext{
*Supported by OTKA Grant PD 75837, and János Bolyai Research Scholarship of the Hungarian Academy of Sciences.

${ }^{\dagger}$ Partly supported by Ministry of Science and Technological Development, Republic of Serbia, and Provincial Secretariat for Science, Province of Vojvodina.
} 


\section{Introduction}

In this paper, we deal with Avoider-Enforcer positional games. For a hypergraph $\mathcal{F}$, the game is played by two players, Avoider and Enforcer. They alternately claim previously unclaimed vertices of $\mathcal{F}$. Avoider starts, and the game ends when all vertices have been claimed. Enforcer wins if Avoider has claimed all vertices of some hyperedge of $\mathcal{F}$. Otherwise Avoider wins. We refer to the vertices of $\mathcal{F}$ as the board, and the hyperedges of $\mathcal{F}$ as the losing sets. The recent book [3] by Beck offers a good overview of the topic of positional games. Here, we study games which are played on the edges of the complete graph on $n$ vertices, that is, the board of $\mathcal{F}$ is always $E\left(K_{n}\right)$.

If we assume that both players play optimally, then each game $\mathcal{F}$ is either an Avoider's win or an Enforcer's win. A significant part of the previous work done in combinatorial game theory (see, e.g., [4]) is devoted to the question: which one of the two players wins a particular game? Here, we go one step further and address a different issue - our hope is to determine not only the winner of a game, but also how fast is he able to win.

For a game $\mathcal{F}$, let $\tau_{E}(\mathcal{F})$ be the smallest integer $t$ such that Enforcer has a strategy to win the game $\mathcal{F}$ in at most $t$ moves. We say that $\tau_{E}(\mathcal{F})=\infty$, if the game is an Avoider's win.

For an Avoider-Enforcer game, this type of question was first raised only recently, by Hefetz et al. in [6], and it was also addressed in [1]. On the other hand, an analogue question for Maker-Breaker games, the more studied Avoider-Enforcer games' counterpart, has been a topic for some time. We mention here the work of Beck [2] and Pekeč [10], who looked at how fast Maker can win the clique game. Chvátal and Erdős [5], and later Hefetz et al. [7] studied the fast winning in Maker-Breaker Hamiltonicity game.

We would like to emphasize that, generally speaking, results on fast winning in positional games have an impact on the whole field, as those results can later be used in analysis of other positional games. Namely, it often happens that an optimal strategy of a player consists of several stages, and in each of them the player wants to complete a task. In that situation, a particular task should not only be performed, but performed fast, i.e., in significantly less moves than the total number of moves at player's disposal an example of this can be found in [9].

\section{$1.1 \quad$ Preliminaries}

The theory behind Avoider-Enforcer games is less developed than the one behind MakerBreaker games. However, when it comes to determining how fast can Enforcer win the game, somewhat unexpected help comes from extremal graph theory.

The extremal number (or Turán number) of a hypergraph $\mathcal{F}$ is defined by $\operatorname{ex}(\mathcal{F})=$ $\max \{|A|: A \subseteq V(\mathcal{F}), A \notin E(\mathcal{F})\}$. As it was shown in [6], if the set of hyperedges of $\mathcal{F}$ is a monotone increasing family of sets, we have

$$
\frac{1}{2} \operatorname{ex}(\mathcal{F})+1 \leqslant \tau_{E}(\mathcal{F}) \leqslant \operatorname{ex}(\mathcal{F})+1
$$

Note that for every game $\mathcal{F}$, we can make the set of hyperedges an increasing family by 
adding all the supersets of the hyperedges. This process changes neither the outcome nor the nature of the game.

Therefore, as soon as we know the extremal number for the game hypergraph, from (1) we get the length of the game squeezed between two values which are roughly a factor of two from each other.

In [1] and [6], the possibilities of Enforcer's fast win for several well-studied positional graph games were analyzed. As it was shown in [1], Avoider can keep his graph planar for as many as $3 n-O(1)$ moves, which is just a constant away from the upper bound derived from (1). Two other basic positional games are looked at in [6]. In the first one, Avoider wants to keep his graph bipartite for as long as possible, where in the second one his goal is to avoid creating a spanning graph. The duration of both games is determined quite precisely in both the first and the second order terms. It turns out that in both cases the values are not an additive constant away from either of the bounds in (1).

\subsection{Our results}

In the present paper, we analyze the duration of the Avoider-Enforcer game for three basic positional games. As we saw in the non-planarity game, in contrast to several other games that were analyzed, Avoider can keep his graph planar for quite a long time, just constant away from the upper bound in (1). This motivated us to analyze another, fairly similar game - a game in which Avoider wants to keep his graph outerplanar for as long as possible. Formally, let $\mathcal{O P}_{n}$ be the hypergraph whose hyperedges are the edge-sets of all non-outerplanar graphs on $n$ vertices. The relation (1) shows that $n \leqslant \tau_{E}\left(\mathcal{O P}_{n}\right) \leqslant 2 n-2$, which leaves $n-1$ possible values for $\tau_{E}$. We manage to narrow down the choice to just five values.

\section{Theorem 1.1}

$$
2 n-7 \leqslant \tau_{E}\left(\mathcal{O} \mathcal{P}_{n}\right) \leqslant 2 n-3
$$

Similarly to the non-planarity game, the duration of the game is just an additive constant away from the upper bound obtained from (1). The common feature of the nonouterplanarity game and the non-planarity game is that in both cases Avoider loses if and only if his graph contains a minor of one of the fixed forbidden graphs. For outerplanarity these forbidden minors are $K_{4}$ and $K_{2,3}$, and for planarity the forbidden minors are $K_{5}$ and $K_{3,3}$. We were curious to further analyze the games of this kind. Hence, we turned our attention to a game where Avoider's goal is to avoid a single forbidden minor in his graph. The forbidden minor is the diamond, that is, $K_{4}$ with one edge missing. We note that [8] deals with a similar game, where Avoider's goal is to avoid claiming a fixed minor. However, the game analyzed there is biased, and the main interest is just the final outcome.

Formally, let $\mathcal{D} \mathcal{F}_{n}$ be the hypergraph whose hyperedges are the edge-sets of all graphs on $n$ vertices that contain a diamond minor. The number of edges in a diamond-free graph is at most $d(n)=\left\lceil\frac{3 n-4}{2}\right\rceil$, as we later show in Lemma 2.2, and from (1) we get 
$\frac{1}{2} d(n)+1 \leqslant \tau_{E}\left(\mathcal{D} \mathcal{F}_{n}\right) \leqslant d(n)+1$. In the following theorem, we reduce this interval to four integers, again an additive constant away from the upper bound.

Theorem 1.2

$$
d(n)-2 \leqslant \tau_{E}\left(\mathcal{D F}_{n}\right) \leqslant d(n)+1 .
$$

We note that diamond-free graphs are sometimes called cactus graphs, and it can be shown that they are outerplanar.

A graph $G$ is called $k$-degenerate, if every subgraph of $G$ has a vertex of degree at most $k$. The degeneracy of a graph is the minimal $k$ such that the graph is $k$-degenerate. Low degeneracy is a common property of planar and outerplanar graphs; their degeneracy is at most 5 and 2 , respectively. It is known that graph degeneracy plays a key role in several other positional games on graphs, see, e.g., [11].

Here, our aim is to study a game in which Avoider's goal is to keep his graph $k$ degenerate, for an integer $k$. In a way, it brings all the mentioned games together, as its family of forbidden graphs, for some values of $k$, contains the aforementioned families of forbidden graphs.

Formally, let $\mathcal{D}_{n}^{k}$ be the hypergraph whose hyperedges are the edge-sets of all graphs on $n$ vertices that are not $k$-degenerate. A $k$-degenerate graph with $n$ vertices can have at most $e(n)=(n-k) k+\left(\begin{array}{c}k \\ 2\end{array}\right)$, and we show that Avoider loses only at the time when he has claimed more than $e(n)$ edges, assuming that $n$ is large enough compared to $k$.

Theorem 1.3 If $k=o(\log n)$, then $\tau_{E}\left(\mathcal{D}_{n}^{k}\right)=e(n)+1$.

Our graph-theoretic notation is standard and follows that of [12]. A matching $M$ of a graph $G$ is called near-perfect if there are at most two $M$-unsaturated vertices in $G$. If $H$ is a graph, we say that a graph $G$ is $H$-free, if $G$ contains no $H$-minor. Throughout the paper, $\log$ stands for the natural logarithm.

Occasionally, we may work with dynamic sets and notations. For instance, $\mathcal{A}$ will denote the set of edges claimed by Avoider during the course of a game. At the start of a game, it is the empty set. If Avoider claims the edge $e$ in his $i$-th move, then we change $\mathcal{A}$ to be $\mathcal{A} \cup e$.

\section{The strategies - fast winning and slow losing}

\subsection{Keeping the graph outerplanar}

Proof of Theorem 1.1. Assume that Avoider claims an edge $u v$ in his first move. Later in the game, if Avoider claims an edge incident to $u v$, say $x v$, then Enforcer claims the edge $x u$ in the next move. This simple pairing strategy enables Enforcer to prevent Avoider from claiming any triangle on the edge $u v$. Therefore, Avoider is unable to claim a maximal outerplanar graph, and loses after at most $2 n-3$ moves.

Next, we show a strategy for Avoider to keep his graph $\mathcal{A}$ outerplanar for $2 n-8$ moves. In his first two moves, Avoider claims two edges of a triangle. We denote the third edge of 
this triangle by $m$. Note that $\mathcal{A} \cup\{m\}$ is a maximal outerplanar graph on three vertices. For most of the game, Avoider maintains the graph $\mathcal{A}$ consisting of a graph one edge short of a maximal outerplanar graph, and some isolated vertices. He achieves that by attaching an isolated vertex to the current outerplanar graph in every pair of consecutive moves.

Throughout the game, we denote the outer face of $\mathcal{A} \cup\{m\}$ by $O_{\mathcal{A}}$. An isolated vertex $v$ in Avoider's graph will be called $b a d$, if for every three consecutive vertices $v_{1}, v_{2}, v_{3}$ on $O_{\mathcal{A}}$ at least one of the edges $v v_{1}, v v_{2}, v v_{3}$ is claimed by Enforcer. Any other isolated vertex of $\mathcal{A}$ is called good. A good vertex can be attached to the current outerplanar graph $\mathcal{A}$ in two Avoider's moves. Namely, if $v_{1}, v_{2}, v_{3}$ are consecutive vertices on $O_{\mathcal{A}}$ and none of the edges $v v_{1}, v v_{2}, v v_{3}$ are claimed, then Avoider can first claim $v v_{2}$, and then one of the edges $v v_{1}, v v_{3}$ in the following move, see Figure 1.

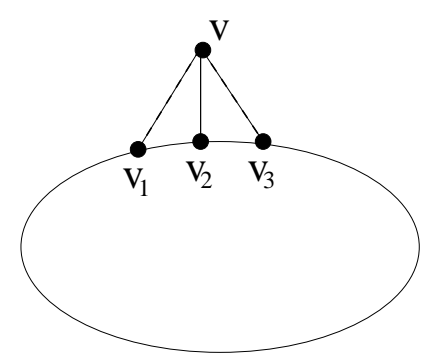

Figure 1: Extension process, which can be performed for good vertices

Let $k$ be the order of the current outerplanar graph $\mathcal{A}$. We claim that the number of bad vertices can never exceed five. Indeed, if there were six bad vertices at some point of the game, then there would be at least $6\left\lceil\frac{k}{3}\right\rceil>2 k-4$ Enforcer's edges. That is more than the total number of edges played by Enforcer until that point.

While $k<\lceil n / 4\rceil$, Avoider always attaches a good vertex of the highest Enforcer's degree. The setup behind this process is similar to the one in the so-called box game. Let $m, s$ and $\ell$ be positive integers. In the box game, in each of the moves, the first player claims $m$ elements of the board, and the second player claims one element of the board. The goal of the first player is to fully claim one of $\ell$ disjoint winning sets of size $s$ each, and his opponent wants to prevent him from doing that. We will make use of the following result.

Theorem 2.1 (Chvátal and Erdös [5]) The first player can win the box game when $s<m \log \ell$.

We want to show that, throughout the game, Avoider can keep Enforcer's maximum degree over all good vertices smaller than $5 \log n$. To do that, we assign a box of size $5 \log n$ to each of the good vertices, and Avoider adopts the role of the second player in the box game. Now, whenever Enforcer claims an edge adjacent to a vertex, we assume that the first player in the box game claimed one element in the box assigned to that 
vertex. In our original game, we group the Enforcer's move in consecutive pairs, and so in each pair of moves four elements will be claimed in the box game. When the second player is to claim an element in a box, then Avoider uses his two moves to attach the good vertex corresponding to that box to the current outerplanar graph. The box is thus removed from the game. Theorem 2.1 with $m=4$ guarantees that the second player in the box game can never fill up a box, and hence, Enforcer's degree will remain below $5 \log n$.

Suppose that $k=\lceil n / 4\rceil$ and there are five bad vertices, $b_{1}, \ldots, b_{5}$. We show how Avoider can reduce the number of bad vertices to four in the two moves that follow. As we have already seen, a bad vertex $v$ is adjacent in Enforcer's graph to at least one of every three consecutive vertices on $O_{\mathcal{A}}$. These vertices of $O_{\mathcal{A}}$ subdivide the edge set of $O_{\mathcal{A}}$ into paths of length at most three, and we will refer to these as blocks. For every $i \in\{1, \ldots, 5\}$ and every edge $e$ on $O_{\mathcal{A}}$, we define $f_{i}(e)$ as the set of edges in the block to which $e$ belongs, in the mentioned subdivision by $b_{i}$. As we have seen, $\left|f_{i}(e)\right|$ is always either 1,2 or 3 .

The number of edges claimed by Enforcer between $b_{i}$ and $V\left(O_{\mathcal{A}}\right)$ is $\sum_{e \in O_{\mathcal{A}}} \frac{1}{\left|f_{i}(e)\right|}$, for $i \in\{1, \ldots, 5\}$. The total number of edges claimed by Enforcer is not less than

$$
\sum_{i=1}^{5} \sum_{e \in O_{\mathcal{A}}} \frac{1}{\left|f_{i}(e)\right|}
$$

On the other hand, we know that Enforcer played at most $2 k-4$ moves in total, and hence,

$$
\sum_{e \in O_{\mathcal{A}}} \sum_{i=1}^{5} \frac{1}{\left|f_{i}(e)\right|} \leqslant 2 k-4 .
$$

Therefore, there exists an edge $e \in O_{\mathcal{A}}$ such that $\sum_{i=1}^{5} \frac{1}{\left|f_{i}(e)\right|}<2$. This can only happen if at least four of $\left|f_{i}(e)\right|, i=1, \ldots, 5$, say the first four, are equal to 3 . Therefore, there has to be an edge $f$ on $O_{\mathcal{A}}$ adjacent to $e$ such that $\{e, f\}$ belong to two of the blocks $f_{i}(e)$, $i=1, \ldots, 5$, say, $f_{1}(e)$ and $f_{2}(e)$.

By $w_{1}, w_{2}, w_{3}$ we denote the three consecutive vertices on $O_{\mathcal{A}}$ with $e=w_{1} w_{2}, f=w_{2} w_{3}$. Since $k=\lceil n / 4\rceil$, there still exists an isolated vertex $u$ in Enforcer's graph. In the following move, Avoider claims the edge $u w_{2}$.

If Enforcer does not claim $u w_{1}$ in his response, Avoider claims it immediately. The vertex $u$ is also on $O_{\mathcal{A}}$ now, and four blocks $f_{i}(e), i=1, \ldots, 4$, are extended to size four in this way. Only two of them can be subdivided by the last two Enforcer's moves. Hence, some $b_{i}$ is not bad any more.

On the other hand, if Enforcer claims $u w_{1}$ in his response, then Avoider claims $u w_{3}$, and similarly as before, blocks $f_{1}(e)$ and $f_{2}(e)$ are extended to size four. Enforcer can subdivide at most one of them in his following move, and the bad vertex corresponding to the other block is not bad any more.

Therefore, after this process, there are at most four bad vertices. As long as $k<n-4$, Avoider keeps attaching good vertices to $\mathcal{A} \cup\{m\}$. Since Enforcer's maximum degree over 
all good vertices will be at $\operatorname{most} 5 \log n$ throughout the game, no other vertex can ever become bad. Finally, when there are only bad vertices left, they are isolated in Avoider's graph. Therefore, Avoider can play at least four more moves without creating a nonouterplanar graph, and the total number of Avoider's moves is at least $2(n-4)-4+4=$ $2 n-8$.

\subsection{Keeping the graph diamond-free}

Recall that a graph is called diamond-free, if it does not contain a diamond as a minor. First, we determine the maximum number of edges in a diamond-free graph on $n$ vertices. A connected graph is called biconnected, if it remains connected after the removal of any vertex. A biconnected component of a graph is a maximal biconnected subgraph. In particular, a single edge might be a biconnected component. Any connected graph decomposes into a tree of biconnected components attached at cut vertices.

Lemma 2.2 If $G$ is a diamond-free graph on $n$ vertices, then the number of edges in $G$ is at most $d(n)$, where $d(n)=\left\lceil\frac{3 n-4}{2}\right\rceil$.

Proof. Let $G$ be a diamond-free graph on $n$ vertices. It is enough to look at connected graphs. Every biconnected component of $G$ has to be either a simple cycle or a single edge. Let us denote the number of biconnected components of $G$ by $b$. We use induction on $b$.

If $b=1$, then $G$ is either an edge or a cycle. In case of an edge, $n=2$ and $\left\lceil\frac{3 n-4}{2}\right\rceil=1$, and the claim holds. In case of a cycle with $n$ vertices, $n \geqslant 3$, we get $\left\lceil\frac{3 n-4}{2}\right\rceil=n+\left\lceil\frac{n-4}{2}\right\rceil \geqslant$ $n$, and the claim holds.

In the induction step, let us assume that the statement holds for connected graphs with at most $b$ biconnected components, $b \geqslant 1$. Now, let $H$ be a connected graph with $b+1$ biconnected components, and let $C$ be a biconnected component of $H$ such that $H-C$ is still connected. As $H-C$ has $b$ biconnected components, the induction hypothesis can be applied. We denote the number of vertices of $H-C$ by $n$, and the number of vertices of $H$ by $n+k$, where $k \geqslant 1$. The number of edges of $H-C$ is at most $\left\lceil\frac{3 n-4}{2}\right\rceil$. There are at most $k+1$ other edges in $H$, if $k \geqslant 2$, and there is one other edge, if $k=1$. Therefore, $d(n+k) \leqslant\left\lceil\frac{3 n-4}{2}\right\rceil+k+1=\left\lceil\frac{3 n+2 k+2-4}{2}\right\rceil \leqslant\left\lceil\frac{3(n+k)-4}{2}\right\rceil$, and the statement holds.

This bound can be attained by gluing together triangles along vertices, adding a single hanging edge if $n$ is even, see Figure 2 .

Next, we show that Avoider can survive in the game for nearly as many moves as the last lemma allows.

Proof of Theorem 1.2. For the lower bound, we give an explicit strategy for Avoider that enables him to play for $d(n)-3$ moves. Before performing a detailed analysis, let us first sketch Avoider's strategy. The game is divided into two phases. In the first phase, Avoider fixes two arbitrary vertices $c_{1}$ and $c_{2}$ and connects them in his first move. Then, by using a pairing strategy, he creates a spanning tree, consisting of two stars centered at $c_{1}$ and $c_{2}$, and the edge $c_{1} c_{2}$. While doing that, Avoider pays attention to certain edge 

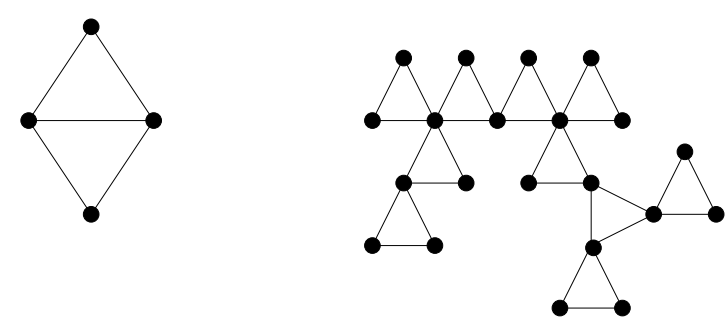

Figure 2: A diamond, and a maximal diamond-free graph on 21 vertices

densities in Enforcer's graph, preparing the ground for the second phase. In the second phase, Avoider claims a large matching on the leaves of each of the stars. In this way, he forms a bunch of edge-disjoint triangles along with a bridge and possibly some hanging edges, that is, a diamond-free graph.

Next, we describe the first phase in detail. Let $c_{1}$ and $c_{2}$ be two vertices, fixed before the game starts. Avoider creates two disjoint stars centered in $c_{1}$ and $c_{2}$. Throughout this phase, we denote the set of vertices adjacent to $c_{i}$ in Avoider's graph by $L_{i}$, for $i=1,2$. The set of vertices that are isolated in Avoider's graph is denoted by $R$.

We list the rules for Avoider's strategy during the first phase. In the first move, he claims the edge $c_{1} c_{2}$. The rest of the rules follow. The first phase ends as soon as Avoider plays a move after which $R=\emptyset$, i.e., we have $V=L_{1} \cup L_{2} \cup\left\{c_{1}, c_{2}\right\}$.

- Whenever Enforcer claims an edge $x c_{i}$, for some $i \in\{1,2\}$, Avoider responds by claiming the edge $x c_{3-i}$,

- If Enforcer claims an edge $u v$, where $u \in L_{i}$, for some $i \in\{1,2\}$, and $v \in R$, then Avoider responds with $v c_{3-i}$,

- If Enforcer claims an edge $u v$, where $u, v \in L_{i}$, for some $i \in\{1,2\}$, then Avoider responds with $w c_{i}$, for arbitrary $w \in R$,

- If Enforcer claims an edge $u v$, with $u, v \in R$, then Avoider responds by claiming $c_{i} u$, where $i$ is arbitrary,

- If Enforcer claims an edge between $L_{1}$ and $L_{2}$, then Avoider responds by claiming $c_{i} u$, where $u$ is any vertex from $R$, and $i$ is arbitrary.

A possible arrangement of edges played is shown in Figure 3.

Let $E_{\mathcal{E}}(X)$ denote the set of edges in Enforcer's graph, induced by $X$. We define the following density measure,

$$
\varrho_{i}=\frac{\left|E_{\mathcal{E}}\left(L_{i}\right)\right|+\left|E_{\mathcal{E}}\left(L_{i}, R\right)\right|}{\max \left\{\left|V\left(L_{i}\right)\right|, 1\right\}}, \text { for } i=1,2 .
$$

We prove that throughout the first phase, after each of his moves, Avoider keeps both $\varrho_{1}$ and $\varrho_{2}$ to be at most 1 . Indeed, the densities from (2) are certainly less than 1 


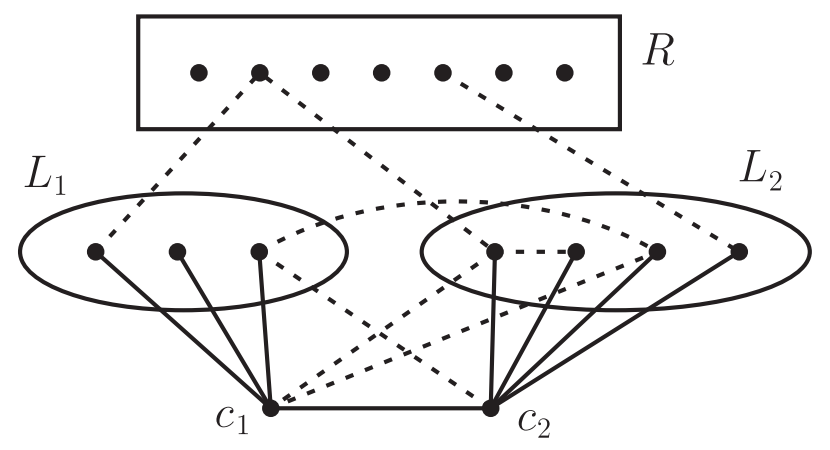

Figure 3: A possible arrangement during the first phase - solid lines represent Avoider's edges, and dashed lines represent Enforcer's edges

after the very first Avoider's move. Next, let us look at an Enforcer's move, and the corresponding Avoider's move. Checking through all of the rules in Avoider's strategy we see that the densities either remain unchanged, or 1 is added to both the numerator and the denominator in (2). Hence, neither of the densities can exceed 1.

We proceed to the second phase, in which Enforcer plays the first move. As we have already mentioned, Avoider's goal is to build a large matching on both $L_{1}$ and $L_{2}$. Throughout this phase, as soon as Avoider claims an edge $v_{1} v_{2} \in L_{i}$, for some $i \in\{1,2\}$, we remove both $v_{1}$ and $v_{2}$ from $L_{i}$. The set of rules for Avoider's strategy in this phase can now be described.

- If Enforcer claims an edge in $L_{i}$, for some $i \in\{1,2\}$, Avoider wants to respond by claiming an edge also in $L_{i}$. Otherwise, Avoider claims an edge in any of the sets $L_{i}, i \in\{1,2\}$.

- Whenever Avoider wants to respond by claiming an edge in $L_{i}, i \in\{1,2\}$, we distinguish two cases:

1. If there is an unclaimed edge in $L_{i}$ that is adjacent to a vertex $m$ with maximum Enforcer's degree in $L_{i}$, Avoider claims it.

2. If there is no unclaimed edge in $L_{i}$ that is adjacent to a vertex $m$ with maximum Enforcer's degree in $L_{i}$, Avoider removes $m$ from $L_{i}$, and then claims an edge following this rule. If after the removal of $m$ there are no unclaimed edges in $L_{i}$, he proceeds by claiming an edge in $L_{j}, j \neq i$.

Following these rules Avoider keeps $\varrho_{i} \leqslant 1$, where $R$ in (2) is now the empty set, for $i=1,2$. If the above condition in 2 . is satisfied, knowing that $\varrho_{i} \leqslant 1$, the new set $L_{i}$ induces at most one Enforcer edge, $e$ say. Avoider's reply is an edge adjacent to $e$, and therefore Enforcer's graph $\mathcal{E}$ becomes empty on $L_{i}$, and remains empty after every of the following Avoider's moves during phase two. That is, case 2. above can happen at most 
once for each $L_{i}$, and whenever it happens Avoider can reach a near-perfect matching in that $L_{i}$.

If case 2. does not occur for $L_{i}, i \in\{1,2\}$, Avoider can follow the algorithm until $\left|L_{i}\right|<4$. If $\left|L_{i}\right| \leqslant 2$, then Avoider has reached a near-perfect matching. The only case when Avoider is possibly stuck is $\left|L_{i}\right|=3$, if after Enforcer's move, $L_{i}$ spans a triangle of Enforcer's edges. We conclude that the total number of vertices in $L_{1} \cup L_{2}$ that are unsaturated by the two matchings is at most six.

As we have already mentioned, phase two is finished, when the matchings of Avoider can not be further extended. By simply counting the edges played, the lower bound from the theorem readily follows.

\section{$2.3 \quad$ Keeping the graph $k$-degenerate}

Recall that $e(n)=(n-k) k+\left(\begin{array}{c}k \\ 2\end{array}\right)$ is the maximal number of edges a $k$-degenerate graph with $n$ vertices can have. Before we prove the theorem, notice an alternative way of defining degeneracy: a graph $G$ is $k$-degenerate if and only if there is a total ordering of $V(G)$ such that any vertex has at most $k$ preceding neighbors in that ordering.

Proof of Theorem 1.3. We exhibit a strategy for Avoider to claim the edges of a maximal $k$-degenerate graph on $n$ vertices in his first $e(n)$ moves. We split the game into two phases.

In the first phase, Avoider wants to create a maximal $k$-degenerate graph on significantly less than $n$ vertices. In the second phase, he gradually attaches all the remaining vertices to that graph.

Let us now describe both phases in detail. The first phase is subdivided into $k$ subphases. In the beginning of the first subphase, Avoider picks a vertex $v_{1}$, and he repeatedly claims edges adjacent to $v_{1}$ until he has claimed $3^{3 k}$ edges. By $V_{1}$ we denote the set of vertices adjacent to $v_{1}$ in Avoider's graph at this point. For $2 \leqslant i \leqslant k$, in the beginning of the $i$-th subphase, Avoider chooses the vertex $v_{i} \in V_{i-1}$ of minimal degree in Enforcer's graph induced on $V_{i-1}$, and connects $v_{i}$ to some $3^{3 k-i+1}$ vertices of $V_{i-1}$. We denote the set of those vertices by $V_{i}$. It remains to show that this can be done, i.e., before the $i$-th subphase there are at least $2 \cdot 3^{3 k-i+1}$ vertices in $V_{i-1}$ such that edges between them and $v_{i}$ are not claimed. This can be seen as follows. The total number of moves played in the first $i-1$ subphases is $\sum_{j=1}^{i-1} 3^{3 k-j+1} \leqslant 3^{3 k+1} / 2$, and the minimum degree in Enforcer's graph taken over all vertices in $V_{i-1}$ is not greater than $3^{3 k+1} /\left|V_{i-1}\right|=3^{i-1}$, implying our claim.

After the end of the first phase, Avoider's graph induced on the set $R=V_{k} \cup$ $\left\{v_{1}, \ldots, v_{k}\right\}$ is a maximal $k$-degenerate graph. During the second phase, the vertices from $V(G) \backslash R$ will be gradually attached to that graph using a pairing strategy. Note that Avoider has claimed some edges between $R$ and $V \backslash R$ already in the first phase.

For every vertex $x \in V(G) \backslash R$, Avoider's first hope is to claim $k$ edges between $x$ and $R$, including the edges played in the first phase. To check if that can be done using a pairing strategy, to each $x$ we assign the following number, 


$$
f(x):=\operatorname{deg}_{\mathcal{A}}(x, R)+\frac{1}{2}\left(|R|-\operatorname{deg}_{\mathcal{E}}(x, R)-\operatorname{deg}_{\mathcal{A}}(x, R)\right) .
$$

Here, $\operatorname{deg}_{\mathcal{A}}(x, R)$ and $\operatorname{deg}_{\mathcal{E}}(x, R)$ stand for the numbers of edges between $x$ and $R$ claimed by Avoider and Enforcer, respectively. By $D$ we denote all vertices in $V(G) \backslash R$ with $f(x) \geqslant k$, and let $F:=(V(G) \backslash R) \backslash D$. Since the total number of edges claimed in the first phase is less than $3^{3 k+1}$, we know that $|F| \leqslant 2 \cdot 3^{3 k+1}<n / 2$.

Now, for every vertex $v \in D$, Avoider will use a simple pairing strategy to claim $k$ edges between $v$ and $R$, also counting the edges he has already claimed in the first phase. To do that, he considers $2\left(k-\operatorname{deg}_{\mathcal{A}}(x, R)\right)$ unclaimed edges between $v$ and $R$, and pairs them up arbitrarily.

For every vertex $v \in F$, Avoider aims at connecting it to a larger set, $R \cup D$. He will again use a simple pairing strategy to claim $k$ edges between $v$ and $R \cup D$. To do that, he considers $2\left(k-\operatorname{deg}_{\mathcal{A}}(x, R)\right)$ unclaimed edges between $v$ and $R \cup D$ and pairs them up arbitrarily.

Avoider's strategy for the second phase is the following. Whenever Enforcer claims

one of the paired edges, Avoider immediately responds by claiming the other one. If Enforcer claims an edge that does not belong to a pair, then Avoider claims an edge in an arbitrary pair, and removes that pair for the rest of the game. As long as Avoider proceeds like this, he will not lose. Indeed, looking at the alternative definition of $k$-degeneracy presented in the beginning of this section, we see that any total ordering $\prec$ in which $\left\{v_{1}, \ldots, v_{k}\right\} \prec V_{k} \prec D \prec F$ verifies that Avoider's graph is $k$-degenerate. When all the pairs are removed he has already claimed a maximal $k$-degenerate graph on $n$ vertices.

\section{Concluding remarks and open problems}

Looking at the Avoider-Enforcer diamond-free game, and the games of non-planarity and non-outerplanarity, we could observe a pattern regarding how long the game lasts. Namely, the number of moves Avoider can survive in those games are all just an additive constant away from the upper bound in (1). We are curious whether this pattern extends to a larger class of forbidden graphs.

Question 3.1 Let $H$ be a fixed graph, and let $\mathcal{F}_{n}^{H}$ be the set of subgraphs of $K_{n}$ that contain an $H$-minor. Is it true that

$$
\tau_{E}\left(\mathcal{F}_{n}^{H}\right)=\operatorname{ex}\left(\mathcal{F}_{n}^{H}\right)+\mathrm{O}(1) ?
$$

Even though our main goal was to prove Theorem 1.3 for constant values of $k$, it turned out that our proof readily holds for all $k=\mathrm{o}(\log n)$. We did not make particular efforts to analyze the same problem for larger values of $k$. Still, we think that it would be interesting to find out for how large $k$, in terms of $n$, the statement of Theorem 1.3 still holds.

Question 3.2 How large can $k=k(n)$ be, so that $\tau_{E}\left(\mathcal{D}_{n}^{k}\right)=e(n)+1$ still holds? 


\section{Acknowledgments}

We would like to thank the anonymous referee for valuable comments and suggestions that improved our paper.

\section{References}

[1] V. Anuradha, C. Jain, J. Snoeyink and T. Szabó, How long can a graph be kept planar?, Electron. J. Combin., 15 (2008), N14.

[2] J. Beck, On positional games, J. Combin. Theory Ser. A, 30 (1981), 117-133.

[3] J. Beck, Tic-Tac-Toe Theory, Cambridge University Press, 2008.

[4] E.R. Berlekamp, J.H. Conway, R.K. Guy, Winning ways for your mathematical plays, Vol. 1-4, AK Peters, Wellesley, 2001.

[5] V. Chvátal and P. Erdős, Biased positional games, Annals of Discrete Math., 2 (1978), $221-228$.

[6] D. Hefetz, M. Krivelevich, M. Stojaković and T. Szabó, Fast winning strategies in Avoider-Enforcer games, Graphs Combin., 25 (2009), 533-544.

[7] D. Hefetz, M. Krivelevich, M. Stojaković and T. Szabó, Fast winning strategies in Maker-Breaker games, J. Combin. Theory Ser. B, 99 (2009), 39-47.

[8] D. Hefetz, M. Krivelevich, M. Stojaković and T. Szabó, Planarity, colorability and minor games, SIAM J. Discrete Math., 22 (2008), 194-212.

[9] M. Krivelevich, The critical bias for the Hamiltonicity game is $(1+o(1)) n / \ln n$, manuscript.

[10] A. Pekeč, A winning strategy for the Ramsey graph game, Combinat. Probab. Comput., 5 (1996), 267-276.

[11] M. Stojaković, Games on Graphs, PhD Thesis, ETH Zurich, 2005.

[12] D.B. West, Introduction to Graph Theory, $2^{\text {nd }}$ edition, Prentice Hall, 2001. 